\title{
Percepción social del cambio climático en estudiantes de Bachillerato Técnico en Jiutepec, Morelos, México
}

Social perception of climate change in students of Technical High School in Jiutepec, Morelos, México

Percepção social da mudança climática de estudantes do Ensino Médio Técnico em Jiutepec, Morelos, México

Indalecio Mendoza Uribe/ Instituto Mexicano de Tecnología del Agua, México/ indalecio mendoza@tlaloc.imta.mx

Olivia Rodríguez López/Instituto Mexicano de Tecnología del Agua, México/ olivia rodriguez@tlaloc.imta.mx

Recibido: 4/2/2021 Aceptado: 8/5/2021 Publicado: 23/6/2021

\section{RESUMEN}

El cambio climático se ha convertido en el principal problema ambiental a nivel planetario, por sus severos impactos en la sociedad y en los ecosistemas. Este trabajo tuvo el propósito de medir la percepción social de dicho fenómeno en estudiantes del Centro de Bachillerato Tecnológico, Industrial y de Servicios 166, en Jiutepec, México. Como método, se realizó una investigación de campo que aplicó una encuesta bajo la escala de Likert, cuyos resultados indicaron que los estudiantes tenían conocimientos sobre el tema y sus efectos negativos. El $80 \%$ de ellos declaró que las actividades humanas contribuyen a dichas alteraciones; a la vez que el $90 \%$ consideró que es responsabilidad de los académicos, científicos, ciudadanos, empresas y gobiernos, el disminuirlas mediante la participación conjunta. Aun cuando se concluye que existe un alto grado de percepción social sobre los actores, causas y efectos principales del cambio climático en los jóvenes de este nivel de enseñanza; todavía no se logra una conciencia plena y un compromiso serio, que fomente la transformación de sus actitudes proambientales en conductas reales como ciudadanos activos y responsables.

Palabras clave: actitudes proambientales, alteraciones climáticas, escala de Likert, participación juvenil 


\begin{abstract}
The climate change had become the main environmental problem at a planetary level because its severe impacts on society and ecosystems. This work had the purpose of measure its social perception in students of the Technological, Industrial and Services High School Center 166 on Jiutepec, México. As a method, a field investigation was applied using a survey under the Likert's scale, which results indicated that the climate change and its effects are known to the students. The $80 \%$ of them declared that human activities are associated with these alterations; also, the $90 \%$ of them said that the responsibility to reduce climate change falls on the joint collaboration between academics, scientists, citizens, companies and governments. Although it is concluded that in high school students there is a high degree of social perception about the main actors, causes and effects of Climate Change; a full awareness and serious commitment that encourages and motivates their active participation as active and responsible citizens haven been achieved yet.
\end{abstract}

Keywords: climatic alterations, Likert's scale, pro-environmental attitudes, youth participation

\title{
RESUMO
}

As mudanças climáticas têm se tornado o principal problema ambiental em nível planetário, devido aos seus graves impactos na sociedade e nos ecossistemas. Diante deste fenômeno, este trabalho teve como objetivo medir a percepção social em alunos do Centro de Bacharelado Tecnológico, Industrial e de Serviços 166, em Jiutepec, México. Quanto a abordagem metodológica, foi realizada uma investigação de campo seguindo critérios de pesquisa na escala Likert, cujos resultados indicaram que os alunos tinham conhecimento sobre o assunto e seus efeitos negativos. No qual, $80 \%$ deles, afirmaram que as atividades humanas contribuem para essas alterações; enquanto $90 \%$, consideram que é responsabilidade de acadêmicos, cientistas, cidadãos, empresas e governos, reduzi-los por meio da participação conjunta. Mesmo concluindo que existe um elevado grau de percepção social sobre os principais atores, causas e efeitos das alterações climáticas por meio dos estudantes. Portanto, a plena consciência e o sério comprometimento ainda não foram alcançados, o que incentiva a transformação de suas atitudes pró-ambientais em comportamentos reais de cidadãos ativos e responsáveis.

Palavras chave: alterações climáticas, escala de Likert, atitudes pró-ambientais, participação juvenil

\section{INTRODUCCIÓN}

La evidencia científica sobre el cambio climático lo posiciona a nivel planetario como el principal problema ambiental, al mostrar sus efectos severos en la sociedad y en los ecosistemas. La Convención Marco 
de las Naciones Unidas sobre el Cambio Climático lo define como el «cambio de clima atribuido directa o indirectamente a la actividad humana, que altera la composición de la atmósfera global y que se suma a la variabilidad natural del clima observada durante períodos de tiempo comparables» (Masson-Delmotte et al., 2018, p. 188). En meteorología se le denomina así a la alteración de las condiciones climáticas predominantes.

Las alteraciones que resultan en el cambio climático son producidas por procesos externos o internos. Dentro de los externos pueden mencionarse la variación de la radiación solar y los parámetros orbitales de la Tierra, los movimientos de la corteza terrestre y la actividad volcánica. Los procesos internos se relacionan con los agentes de forzamiento radiativo. Estos varían de forma natural y por los denominados gases de efecto invernadero provenientes de la actividad humana como el dióxido de carbono, el óxido nitroso y el metano, entre otros. Los cambios en la concentración de estos gases modifican la capacidad de absorción, dispersión y emisión de la radiación dentro de la atmósfera y en la superficie terrestre. De ahí que, el calentamiento global es el aumento de la temperatura de la atmósfera terrestre y los océanos, considerándose como agente del cambio climático por sus efectos en el ambiente (Miranda, 2018).

Desde el punto de vista científico, los impactos son: el aumento de la temperatura promedio del planeta y del vapor de agua en la atmósfera terrestre, que propicia la ocurrencia de precipitaciones más extremas e impredecibles; cambios en los patrones del clima, que provocan una disminución del rendimiento de las cosechas; aumento del número e intensidad de los desastres naturales como las inundaciones y sequías; cambios en la composición y distribución de los bosques; y el aumento de enfermedades infecciosas (García, 2011; Reyna-Fabian et al., 2018; Bastidas y Hernández, 2019; Restrepo-Betancur et al., 2019). En cuanto al impacto social se puede mencionar que el aumento de la temperatura presenta mayores riesgos para erradicar la pobreza, reducir las desigualdades y asegurar el bienestar humano. Esto se debe a que afecta de forma desproporcionada a las poblaciones desfavorecidas y vulnerables, a través de la seguridad alimentaria, el aumento de los precios de los alimentos, la pérdida de ingresos y de oportunidades de subsistencia, los efectos adversos en la salud y el desplazamiento de la población (Ojeda, 2018).

Aún a principios del siglo XXI, la conciencia del hombre y su comportamiento ante los problemas ambientales parecen ser mínimos. El cambio climático no es la excepción, ya que gran parte de su atención se enfoca más en los aspectos físicos y naturales que los originan, así como en las responsabilidades políticas y económicas de los países y empresas que contribuyen a las emisiones de gases de efecto invernadero. Los aspectos sociales, en cambio, son menos tratados. Las comunidades que son vulnerables a amenazas geográficas específicas son tan importantes como 
aquellas que son inermes, debido a las deficiencias en su infraestructura física, lo que las hace particularmente endebles a las inundaciones, olas de calor o la escasez de agua (Clayton, 2019).

En la esfera de la opinión pública y de la percepción social, el cambio climático comenzó a ser considerado como un problema dentro de la preocupación por el ambiente en los años ochenta; pero no fue hasta la década siguiente que tomó notoriedad. La Conferencia de las Naciones Unidas sobre el Medio Ambiente y el Desarrollo celebrada en Río de Janeiro el 12 de junio de 1992 constituyó un momento importante en la aspiración de lograr un equilibrio justo entre las necesidades económicas, sociales y ecológicas. La reunión conocida como Cumbre de la Tierra contó con la participación de 108 jefes de Estado, quienes tuvieron parte en las sesiones plenarias. Durante la cumbre se aprobaron documentos en torno al cuidado del ambiente que dieron origen a nuevas políticas (Gädicke et al., 2017). Sin embargo, para enfrentar los efectos del cambio climático no basta con adoptar políticas de adaptación y mitigación. Es preciso también contar con altos niveles de comprensión, consenso y trabajo participativo entre la ciudadanía, las empresas y las diferentes instancias del poder público, si se quieren trascender las fronteras y actuar desde lo local, regional y global (Cerati y Queiroz, 2016 y Quintana, 2016).

El desconocimiento de la población sobre estos tópicos es una situación que debe ser considerada de forma urgente. De ahí que sea necesario establecerse planes $y$ programas educativos que conduzcan a la toma de conciencia y la sensibilización de la población, que promuevan el desarrollo de valores ambientales y el cambio de actitudes en las personas. De esta manera se fomenta su participación en acciones que contribuyan a controlar la emisión de gases de efecto invernadero, y a disminuir sus secuelas (González, 2016). Ello requiere entender que el grado de conocimiento y la percepción social del problema. Los diferentes sectores de la población son fundamentales para diseñar políticas públicas de adaptación y mitigación que converjan con los saberes, creencias y efectos sentidos de los grupos sociales, en todas las escalas geográficas (Corona-Jiménez, 2018).

En psicología, la percepción se define como:

el proceso cognitivo de la conciencia que consiste en el reconocimiento, interpretación y significación para la elaboración de juicios en torno a las sensaciones obtenidas del ambiente físico y social, en el que intervienen otros procesos psíquicos entre los que se encuentran el aprendizaje, la memoria y la simbolización. (Vargas, 1994, p. 48)

La percepción ambiental implica el proceso de conocer el ambiente físico inmediato a través de los sentidos, a diferencia del conocimiento ambiental que comprende el almacenamiento, la organización y la reconstrucción de imágenes de las características ambientales que no están a la vista en el momento. La relevancia de estudiar la percepción ambiental radica en el hecho de que es el comportamiento 
del ser humano lo que afecta y degrada el ambiente (Martínez-Soto, 2004). Por su parte la aptitud social de la percepción ambiental es una capacidad adaptativa que puede ser construida, potenciada o inhibida por factores socioculturales e individuales (Orozco et al., 2020).

Es por ello que el cambio climático es uno de los mayores desafíos ambientales que enfrenta la humanidad (Soares et al., 2018). De ahí que las acciones de adaptación y mitigación serán asumidas en la medida en que se perciba dicha alteración del clima. El objetivo de la investigación que se presenta fue medir la percepción social de dicho fenómeno en estudiantes del Centro de Bachillerato Tecnológico Industrial y de Servicios 166, en Jiutepec, México, quienes se incorporarán al sector productivo o continuarán sus estudios a corto y mediano plazo.

\section{MATERIALES Y MÉTODOS}

La metodología tuvo un enfoque cuantitativo. Se basó en una investigación de campo, que utilizó como instrumento de recolección de datos una encuesta de carácter descriptivo. La misma estuvo conformada por reactivos con respuestas cerradas en una escala de Likert, con el fin de identificar el grado de percepción de los estudiantes sobre el tema del cambio climático (actores, causas y efectos).

La escala de Likert es un instrumento estructurado de recolección de datos primarios, cuyo formato implica una serie de respuestas calificadas en un nivel de acuerdo o desacuerdo con cada uno de los elementos declarados (Morillo et al., 2019). La estimación otorga al sujeto una puntuación por ítem y una puntuación total que permite precisar, en menor o mayor grado, la presencia del atributo o variable investigada. Por ese motivo se centra en la persona y enfatiza sus diferencias individuales (Vázquez-Guzmán, 2018).

Para medir la actitud o la percepción social del objeto de estudio es preciso definir una escala de medida basada en una serie de indicadores. Estos se seleccionaron de forma cuidadosa de modo que constituyan un criterio válido, fiable y preciso. Cada ítem puede ser una frase, juicio, proposición, reactivo o sentencia que exprese una idea positiva o negativa sobre aquel. Existen tres criterios fundamentales para la confección de los ítems (Fernández, 1982):

1. Deben facilitar respuestas relacionadas con el fenómeno de estudio (variable medida), aunque dicha relación no tiene por qué ser necesariamente manifiesta.

2. Deben declarar no solo las dos posturas extremas, sino también graduar las intermedias. A medida que la escala gane en sensibilidad, también ganará en precisión.

3. Tienen que ser fiables y seguros. La fiabilidad con frecuencia se logra a costa de precisión. Cuanto más refinada es una medición, más probable es que en dos medidas repetidas se obtengan puntuaciones distintas.

Con relación al número de alternativas, algunos autores recomiendan que deberían tener cinco opciones de respuesta, dentro 
de estas un punto medio neutral para acentuar la confiabilidad del instrumento (Matas, 2018). Además, no existen ventajas psicométricas en escalas con más de seis opciones de respuesta (Simms et al., 2019).

En el presente estudio se formularon 10 ítems concretos para medir la percepción social del cambio climático. Los cuatro primeros reactivos se relacionan con las evidencias o efectos, los siguientes tres con las causas y los últimos tres con los actores. A cada pregunta le fue asociada un total de cinco posibles respuestas cerradas, a las que se les asignó un peso entre 1 y 5 . El valor 1 indica muy baja percepción y el valor 5 muy alta percepción sobre la existencia del cambio climático, sus actores, causas y efectos principales en el entorno de los encuestados. Los rangos intermedios mostraron disímiles grados de percepción.
Para evitar el sesgo de respuesta de consentimiento, los enunciados se formularon en forma de pregunta en orden correcto para evitar confusiones e incrementar la eficacia del instrumento. Las opciones de respuesta incluyeron palabras descriptivas de fácil comprensión, unipolarmente; es decir, midieron los diferentes niveles de percepción. Las respuestas de cada ítem no fueron las mismas, pero mantuvieron la regla de medición bajo la interpretación siguiente: a mayor puntaje, más positiva y favorable fue la actitud hacia el proceso de investigación; a menor puntaje, la actitud fue más negativa y desfavorable. Los puntajes intermedios expresaron una actitud medianamente positiva, neutra o medianamente negativa. En la tabla 1 se presentan los 10 ítems del cuestionario aplicado.

Tabla 1. Ítems del cuestionario

\begin{tabular}{|c|c|c|c|}
\hline No & Ítem & Respuestas & Peso \\
\hline \multirow{5}{*}{1} & \multirow{5}{*}{$\begin{array}{l}\text { ¿En los últimos años ha } \\
\text { aumentado la temperatura? }\end{array}$} & Muy en desacuerdo & 1 \\
\hline & & Algo en desacuerdo & 2 \\
\hline & & Ni de acuerdo ni en desacuerdo & 3 \\
\hline & & Algo de acuerdo & 4 \\
\hline & & Muy de acuerdo & 5 \\
\hline \multirow{5}{*}{2} & \multirow{5}{*}{$\begin{array}{l}\text { ¿En los últimos años han } \\
\text { aumentado las Iluvias extremas? }\end{array}$} & Muy en desacuerdo & 1 \\
\hline & & Algo en desacuerdo & 2 \\
\hline & & Ni de acuerdo ni en desacuerdo & 3 \\
\hline & & Algo de acuerdo & 4 \\
\hline & & Muy de acuerdo & 5 \\
\hline \multirow{5}{*}{3} & \multirow{5}{*}{$\begin{array}{l}\text { ¿En los últimos años han } \\
\text { aumentado las enfermedades? }\end{array}$} & Muy en desacuerdo & 1 \\
\hline & & Algo en desacuerdo & 2 \\
\hline & & Ni de acuerdo ni en desacuerdo & 3 \\
\hline & & Algo de acuerdo & 4 \\
\hline & & Muy de acuerdo & 5 \\
\hline \multirow{5}{*}{4} & \multirow{5}{*}{$\begin{array}{l}\text { ¿En los últimos años los } \\
\text { fenómenos meteorológicos } \\
\text { extremos se han intensificado } \\
\text { (huracanes, frentes fríos, sequía, } \\
\text { etc.)? }\end{array}$} & Muy en desacuerdo & 1 \\
\hline & & Algo en desacuerdo & 2 \\
\hline & & Ni de acuerdo ni en desacuerdo & 3 \\
\hline & & Algo de acuerdo & 4 \\
\hline & & Muy de acuerdo & 5 \\
\hline
\end{tabular}




\begin{tabular}{|c|c|c|c|}
\hline \multirow{5}{*}{5} & \multirow{5}{*}{$\begin{array}{l}\text { ¿Qué factores han causado el } \\
\text { aumento de temperatura en los } \\
\text { últimos años? }\end{array}$} & $\begin{array}{l}\text { No estoy seguro de que exista un aumento de la } \\
\text { temperatura }\end{array}$ & 1 \\
\hline & & Es una variación del clima normal (cíclica) & 2 \\
\hline & & Causas naturales (radiación solar, fumarolas, etc.) & 3 \\
\hline & & Uso de combustibles fósiles & 4 \\
\hline & & $\begin{array}{l}\text { Todas las actividades antropogénicas (agricultura, } \\
\text { industria, minería, transporte, etc.) }\end{array}$ & 5 \\
\hline \multirow{5}{*}{6} & \multirow{5}{*}{$\begin{array}{l}\text { ¿Los gases de efecto } \\
\text { invernadero propician el } \\
\text { calentamiento global? }\end{array}$} & Muy en desacuerdo & 1 \\
\hline & & Algo en desacuerdo & 2 \\
\hline & & Ni de acuerdo ni en desacuerdo & 3 \\
\hline & & Algo de acuerdo & 4 \\
\hline & & Muy de acuerdo & 5 \\
\hline \multirow{5}{*}{7} & \multirow{5}{*}{$\begin{array}{l}\text { ¿El calentamiento global es un } \\
\text { aspecto del cambio climático? }\end{array}$} & Muy en desacuerdo & 1 \\
\hline & & Algo en desacuerdo & 2 \\
\hline & & Ni de acuerdo ni en desacuerdo & 3 \\
\hline & & Algo de acuerdo & 4 \\
\hline & & Muy de acuerdo & 5 \\
\hline \multirow{5}{*}{8} & \multirow{5}{*}{$\begin{array}{l}\text { ¿En qué medida consideras que } \\
\text { el aumento de la población } \\
\text { influye en el cambio climático? }\end{array}$} & $\begin{array}{l}\text { El número de habitantes no influye en el cambio } \\
\text { climático }\end{array}$ & 1 \\
\hline & & $\begin{array}{l}\text { Demanda de mayor cantidad de alimentos y uso de } \\
\text { energía }\end{array}$ & 2 \\
\hline & & $\begin{array}{l}\text { Aumento de la agricultura, urbanización y } \\
\text { deforestación }\end{array}$ & 3 \\
\hline & & $\begin{array}{l}\text { Aumento de la industria, minería, construcción y } \\
\text { transporte }\end{array}$ & 4 \\
\hline & & $\begin{array}{l}\text { El cambio climático está ligado directamente a } \\
\text { todas las acciones humanas }\end{array}$ & 5 \\
\hline \multirow{5}{*}{9} & \multirow{5}{*}{$\begin{array}{l}\text { ¿Quiénes son los responsables } \\
\text { de disminuir el calentamiento } \\
\text { global? }\end{array}$} & Ciudadanos & 1 \\
\hline & & Académicos y científicos & 2 \\
\hline & & Empresas & 3 \\
\hline & & Gobierno & 4 \\
\hline & & Todos los anteriores & 5 \\
\hline \multirow{5}{*}{10} & \multirow{5}{*}{$\begin{array}{l}\text { ¿Cómo ciudadano puedes } \\
\text { contribuir a disminuir el } \\
\text { calentamiento global? }\end{array}$} & Muy en desacuerdo & 1 \\
\hline & & Algo en desacuerdo & 2 \\
\hline & & Ni de acuerdo ni en desacuerdo & 3 \\
\hline & & Algo de acuerdo & 4 \\
\hline & & Muy de acuerdo & 5 \\
\hline
\end{tabular}

Fuente: Elaboración propia.

Las encuestas se aplicaron a 100 estudiantes del Centro de Bachillerato Tecnológico Industrial y de Servicios 166 durante el mes de mayo de 2019. La institución está ubicada en el municipio de Jiutepec, Morelos, México. Las edades oscilaron entre los 16 y 18 años. La muestra fue aleatoria y se seleccionaron 50 hombres y 50 mujeres de las carreras técnicas de Administración de Recursos Humanos, Clínico Laboratorista y Programación, del $2 .^{\circ}, 4 .^{\circ}$ y $6 .^{\circ}$ semestre. La encuesta incluyó de forma suplementaria 
y opcional los campos correspondientes a nombre, edad, sexo, carrera, semestre, fecha y comentarios adicionales.

Para cuantificar el grado de percepción del cambio climático se determinó la puntuación obtenida por cada participante, según las alternativas de respuesta seleccionadas. La puntuación máxima teórica fue de 50 y la mínima de 10 . Se determinaron cinco niveles de percepción: muy poco, poco, moderado, alto y muy alto. Para obtener el rango de valores de cada nivel, se aplicó el análisis de quintiles mediante cuatro pasos. Adicionalmente, se agregó un quinto paso para validar la confiabilidad de la escala de Likert aplicada. A continuación, se describe el procedimiento:

\section{Paso 1. Ordenamiento de los datos}

Los posibles valores obtenidos se ordenaron bajo la escala de Likert en la muestra X (tabla 2).

Tabla 2. Valores ordenados de los posibles valores de la sumatoria de los ítems

\begin{tabular}{|c|c|c|c|c|c|c|c|c|c|c|c|c|}
\hline$X_{1}$ & $X_{2}$ & $X_{3}$ & $X_{4}$ & $X_{5}$ & $X_{6}$ & $x_{7}$ & $X_{8}$ & $X_{9}$ & $X_{10}$ & $X_{11}$ & $X_{12}$ & $\mathrm{X}_{13}$ \\
\hline 10 & 11 & 12 & 13 & 14 & 15 & 16 & 17 & 18 & 19 & 20 & 21 & 22 \\
\hline$X_{14}$ & $X_{15}$ & $X_{16}$ & $X_{17}$ & $\mathrm{X}_{18}$ & $X_{19}$ & $X_{20}$ & $X_{21}$ & $X_{22}$ & $X_{23}$ & $X_{24}$ & $X_{25}$ & $\overline{X_{26}}$ \\
\hline 23 & 24 & 25 & 26 & 27 & 28 & 29 & 30 & 31 & 32 & 33 & 34 & 35 \\
\hline$X_{27}$ & $X_{28}$ & $X_{29}$ & $x_{30}$ & $X_{31}$ & $X_{32}$ & $X_{33}$ & $X_{34}$ & $X_{35}$ & $X_{36}$ & $X_{37}$ & $X_{38}$ & $\overline{X_{39}}$ \\
\hline 36 & 37 & 38 & 39 & 40 & 41 & 42 & 43 & 44 & 45 & 46 & 47 & 48 \\
\hline$X_{40}$ & $\mathrm{X}_{41}$ & & & & & & & & & & & \\
\hline 49 & 50 & & & & & & & & & & & \\
\hline
\end{tabular}

Fuente: Elaboración propia.

Paso 2. Definición del número total de elementos de la muestra

El total de elementos de la muestra estuvo compuesto por los posibles valores derivados de la sumatoria de los 10 ítems, con un valor inicial de 10 y final de $50(n=41)$.

\section{Paso 3. Posicionamiento y rango de los quintiles}

Para calcular la posición de cada quintil se utilizó la ecuación 1.

$$
p=\frac{j n}{5}
$$

donde:

$p$ : posición que ocupa el quintil

$j:$ número de quintil

$n$ : número total de datos

Para los valores de $p$ que no tuvieron decimales, el valor del quintil $j$ correspondió al de la muestra $(X)$ en la posición $p$. Por otro lado, para los resultados de $p$ que incluyeron decimales, la posición se ajustó redondeando la media entre los valores de $p$ y $p+1$. En la tabla 3 se presenta la posición y rango de los quintiles. 
Tabla 3. Posición y rango de los quintiles

\begin{tabular}{ccccccc}
\hline $\begin{array}{c}\text { Quintil } \\
\left(\mathbf{Q}_{\mathbf{j}}\right)\end{array}$ & $\begin{array}{c}\text { Nivel de percepción } \\
\text { de cambio climático }\end{array}$ & \multicolumn{2}{c}{ Posición del quintil $(\mathbf{p})$} & & $\begin{array}{c}\text { Valor de la } \\
\text { muestra }\left(\mathbf{X}_{\mathbf{p}}\right)\end{array}$ & $\begin{array}{c}\text { Rango de valores } \\
\text { del quintil }\end{array}$ \\
\hline $\mathrm{Q}_{1}$ & Muy poco & 8.2 & 9 & 18 & $10-18$ \\
$\mathrm{Q}_{2}$ & Poco & 16.4 & 17 & 26 & $19-26$ \\
$\mathrm{Q}_{3}$ & Moderado & 24.6 & 25 & 34 & $27-34$ \\
$\mathrm{Q}_{4}$ & Alto & 32.8 & 33 & 42 & $35-42$ \\
$\mathrm{Q}_{5}$ & Muy alto & 41.0 & 41 & 50 & $43-50$ \\
\hline
\end{tabular}

Fuente: Elaboración propia.

Paso 4. Cálculo de percepción social del cambio climático individual y muestral

Se realizó la sumatoria de los ítems por encuestado para determinar su nivel de percepción sobre el cambio climático. Para obtener la estimación de toda la muestra se efectuó un promedio simple de la calificación obtenida por todos los individuos.

\section{Paso 5. Confiabilidad de la escala de Likert}

Todo instrumento de investigación puede ser evaluado para determinar su grado de confiabilidad. El coeficiente Alfa de Cronbach es un indicador de confiabilidad de escalas psicométricas, y corresponde a una medida de la consistencia interna que presentan los reactivos. Sus valores oscilan entre 0 y 1 : mientras más se acerque el instrumento a la unidad, la confiabilidad de la escala es mayor y se tiene evidencia de su homogeneidad; es decir, que los ítems están apuntando en la misma dirección (Aguilar y Sánchez, 2018; Blanco et al., 2019).

Al respecto, el coeficiente Alfa de Cronbach puede interpretarse como la medida unidimensional de una escala, por lo cual es de utilidad para medir la consistencia de una escala de Likert como la empleada en este estudio. Este se obtiene a partir de la covarianza (intercorrelaciones) entre los ítems, la varianza total de la escala y el número de reactivos que la conforman (ecuación 2). Envtre los trabajos que han aplicado y validado este coeficiente, se ubican los de Hernández y Pascual (2018) y RodríguezRodríguez y Renguant-Álvarez (2020).

$$
\alpha=\frac{k}{k-1}\left[1-\frac{\sum_{i=1}^{k} \sigma_{Y_{i}}^{2}}{\sigma_{X}^{2}}\right]
$$

donde:

$\alpha$ : coeficiente Alfa de Cronbach

k: número de ítems

$\sigma_{Y_{i}}^{2}$ : varianza del ítem $i$

$\sigma_{X}^{2}$ : varianza de los valores totales observados

Para la interpretación de la magnitud de confiabilidad del coeficiente Alfa de Cronbach. En este estudio se utilizó como referencia la propuesta por Ruiz (2013), quien define cinco niveles de confiabilidad tal y como se presenta en la tabla 4. 
Tabla 4. Magnitud de confiabilidad según el coeficiente Alfa de Cronbach

\begin{tabular}{cc}
\hline Rangos de $\boldsymbol{\alpha}$ & Magnitud de confiabilidad \\
\hline 0.81 a 1.00 & Muy alta \\
0.61 a 0.80 & Alta \\
0.41 a 0.60 & Moderada \\
0.21 a 0.40 & Baja \\
0.01 a 0.20 & Muy baja \\
\hline
\end{tabular}

Fuente: Ruiz (2013).

La aplicación del coeficiente sobre los datos de la encuesta dio como resultado el valor de 0.6962. De acuerdo con la escala de la tabla 4 este indica que la escala de Likert aplicada tiene una confiabilidad alta. A continuación, se desglosa la solución de la ecuación 2.

$$
\begin{gathered}
\alpha=\frac{100}{100-1}\left[1-\frac{11.0464}{35.5446}\right] \\
\alpha=\frac{100}{99}[1-0.3108] \\
\alpha=1.0101[0.6892] \\
\alpha=0.6962
\end{gathered}
$$

\section{RESULTADOS}

Los resultados arrojaron que, desde una perspectiva general, el cambio climático y sus efectos no es un tema ajeno a los estudiantes de bachillerato técnico del municipio de Jiutepec. Los encuestados reconocieron que el aumento de la temperatura, el aumento de las enfermedades y la intensificación de los eventos meteorológicos extremos están asociados al cambio climático.

Con relación a las lluvias, solo el $60 \%$ de los encuestados afirmó que se han vuelto más extremas. Esto se explica por la alta variabilidad que presenta el comportamiento natural de la lluvia (Gil-Guirado, 2017), lo que complica la percepción cualitativa entre diferentes años, sobre todo, por la juventud que empieza a tomar conciencia e importancia de su entorno. Sobre las causas que ocasionan el cambio climático, para el $80 \%$ de los estudiantes fue evidente que las actividades humanas, también conocidas como actividades antropogénicas, están asociadas directamente a con las causas y efectos ambientales que originan dichas alteraciones del clima. Respecto a los actores, para más del $90 \%$ de los jóvenes, la responsabilidad de disminuir el calentamiento global recayó en la participación conjunta entre académicos, científicos, ciudadanos, empresas y gobiernos. No obstante, el $5 \%$ señaló que los ciudadanos no pueden contribuir a ello. El $8 \%$ indicó que es en la ciudadanía donde debe estar todo el compromiso para mitigarlo.

La estadística sobre la percepción individual por quintil dio como resultados que no existen estudiantes con muy poca percepción del cambio climático $\left(\mathrm{Q}_{1}\right)$. El $3 \%$ tiene poca percepción $\left(\mathrm{Q}_{2}\right)$, el $8 \%$ una percepción moderada $\left(\mathrm{Q}_{3}\right)$, el $27 \%$ una alta percepción $\left(\mathrm{Q}_{4}\right) \mathrm{y}$, sorpresivamente, el $62 \%$ una percepción muy alta sobre los efectos, causas y actores principales $\left(\mathrm{Q}_{5}\right)$. La percepción promedio de la muestra arrojó un valor de 42 puntos de los 50 posibles. De acuerdo con la tabla 3 este valor se ubica en el límite superior del $Q_{4}$. Lo que 
demuestra que el nivel de conocimientos y percepción de toda la muestra es alto, en términos generales. Los resultados por género no manifestaron gran diferencia en la percepción social del cambio climático. De un total de 4247 puntos obtenidos en la sumatoria de los 10 ítems de las 100 encuestas, el $51 \%$ lo obtuvieron las mujeres, mientras que el $49 \%$ restante se le atribuyó a los hombres.

En la figura 1 se presentan los resultados del cuestionario.

Figura 1. Resultados de la encuesta por ítem

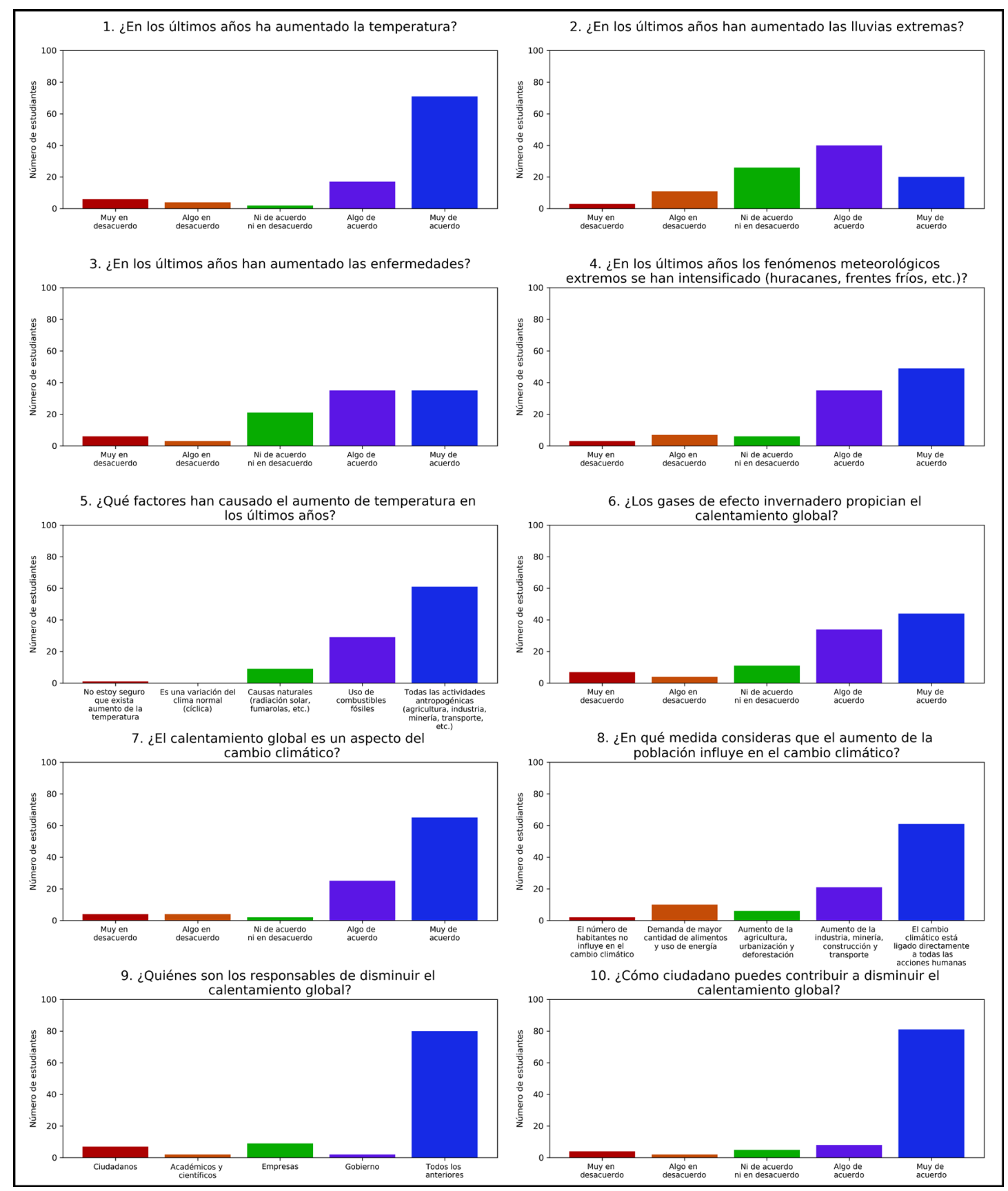

Fuente: Elaboración propia. 


\section{DISCUSIÓN}

Como se pudo evidenciar, la mayor parte de los encuestados conocen sobre el cambio climático, sus causas y efectos. Estos resultados son similares a los expuestos por Olmos et al. (2013), quienes concluyen que, en general, la población de estudio se percata y tiene el conocimiento empírico de los cambios en el ambiente y sus afectaciones, tanto en aspectos económicos como sociales. De igual forma Espejel y Flores (2015) evidenciaron que los estudiantes del Centro de Bachillerato Tecnológico Industrial y de Servicios 212 (Tetla de la Solidaridad, Tlaxcala), tiene conocimiento de la temática de calentamiento global, estos no son suficientes. Lo anterior se debe a que el personal docente no está capacitado para impartir a profundidad este tópico y fomentar conductas positivas.

Los elementos anteriores destacan la necesidad de la incorporación y jerarquización de estos temas en la formación de los docentes de la educación básica y subsiguientes, para que las nuevas generaciones no solo tengan conocimiento y una alta percepción del cambio climático, sino que además sean actores activos desde temprana edad en acciones de mitigación y reducción de las causas que lo generan. Es por ello que el papel de las escuelas toma relevancia como entidad social encargada de la transmisión de los valores. Lo anterior permite a los estudiantes sostener actitudes y comportamientos responsables y amigables con el ambiente, como condición para poder construir proyectos sociales prósperos y sostenibles (Paula-Acosta et al., 2019).
Del mismo modo, Cruz y Páramo (2020) consideran necesario aumentar y afinar el conocimiento básico y aplicado sobre la percepción social para poder diseñar, aplicar y legitimar acciones educativas en el marco de las políticas de respuesta a la crisis climática. El éxito de las medidas de mitigación y adaptación de este fenómeno dependen de la participación activa del conjunto poblacional más amplio. Lo que hace necesario emprender enormes esfuerzos en educación ambiental y comunicación mediante todos los medios disponibles.

Por otro lado, los encuestados manifestaron interés en el tema y disposición para participar en acciones de mitigación. Reacciones similares se observan en otros trabajos como el de Corona-Jiménez (2018). Estas actitudes proambientales son definidas como el conjunto de sentimientos favorables o desfavorables que se tienen hacia alguna característica del medio físico o hacia algún problema relacionado con este. No obstante, existe una brecha entre el decir y el hacer, porque las actitudes subsisten previa a la emisión de las conductas. Tener la intención no significa que estas se realicen finalmente (Páramo, 2017). La correlación entre actitudes proambientales y conductas ecológicamente responsables es, en general, muy baja (Andrade y González, 2019).

En este estudio, los resultados por género no arrojaron gran diferencia en la percepción de hombres y mujeres sobre el cambio climático. A diferencia de lo expuesto por Olmos-Martínez et al. (2016) en su presentación de la percepción, opinión $y$ acciones de estudiantes universitarios sobre cambio climático. Al destacar ciertas 
diferencias entre las opiniones de hombres y mujeres sobre las causas que lo ocasionan. Pese a ello ambos géneros concordaron en que el impacto principal y nivel de afectación es para toda la sociedad.

De acuerdo con Álvarez-Vergnani (2019), la forma menos riesgosa, menos costosa y más productiva para alcanzar el bien común y disminuir los efectos del cambio climático es el ejercicio de la ciudadanía activa y responsable. Se considera que la percepción social identificada en este estudio puede aplicarse en el fomento de jóvenes activos que adopten acciones de mitigación que se generan e impactan al ambiente. Entre las acciones que se pueden realizar desde la ciudadanía, se encuentran: ahorro de energía mediante la disminución del uso de aires acondicionados y desconexión de aparatos electrónicos en desuso; reducir la emisión de dióxido de carbono a través de la reducción del uso de vehículos con motores de combustión interna y reducir el volumen de residuos por medio de tres pasos: reducir, reciclar y reutilizar; entre otras.

\section{CONCLUSIONES}

La aplicación de la escala de Likert permitió medir de forma cuantitativa la percepción del cambio climático en estudiantes de bachillerato con especialidad técnica. Los encuestados pudieron matizar su opinión de manera clara y sencilla, mediante cinco posibles respuestas por ítem con una puntuación unidireccional. Su confiabilidad de la escala Likert empleada se verificó a través del coeficiente Alfa de Cronbach, que arrojó un valor de 0.6962, interpretado como alto. Con base en este resultado, se recomienda la utilización de la escala para investigaciones relacionadas con la percepción social de fenómenos naturales.

Los estudiantes del Centro de Bachillerato Tecnológico Industrial y de Servicios 166 tienen un alto grado de percepción social sobre los actores, causas y efectos del cambio climático. Además, reconocen la importancia de su participación como ciudadanos y próxima fuerza productiva. En contraste, el problema real corresponde al hecho de que no existen una conciencia plena y un compromiso serio que fomente y motive la participación activa de todos los actores, donde las actitudes proambientales se resumen en buenas intenciones y no en actividad práctica. Por tanto, se considera imperante la transformación hacia una ciudadanía activa y responsable. Si bien las nuevas generaciones no son las responsables del cambio climático, el rol y compromiso que adopten los estudiantes de bachillerato será determinante para revertir, o por lo menos mitigar, el problema existente.

\section{REFERENCIAS BIBLIOGRÁFICAS}

Álvarez-Vergnani, C. (2019). Participación ciudadana: retos para una ciudadanía activa ante el cambio climático. Cuadernos de Investigación UNED, 11(1), S78-S88. https://doi.org/10.22458/uri.v11i1.2325

Aguilar, C. M. y Sánchez, G. I. (2018). Construcción y validación de un instrumento para valorar desempeños pedagógicos de estudiantes en formación inicial. Revista Educación, 42(1), 54-68. http://dx.doi.org/10.15517/revedu.v42i1.22728 
Andrade, J. A. y González, J. (2019). Relación entre actitudes pro-ambientales y conocimientos ecológicos en adolescentes con relación al entorno rural o urbano que habitan. Revista Kavilando, 11(1), 105-118. https://bit.ly/2QRe9UT

Bastidas, G. A. y Hernández, R. (2019). Cambio climático algunos aspectos a considerar para la supervivencia del ser vivo: revisión sistemática de la literatura. Revista Cuidarte, 10(3), e664. http://dx.doi.org/10.15649/cuidarte.v10i3.664

Blanco, Y., Torres, J. M., Bernal, E. J. y García, A. S. (2019). Cuestionario para caracterizar las lesiones no intencionales en niños de uno a cuatro años. MediSur, 17(2), 221-229. https://bit.ly/3xQIvY4

Cerati, T. M. y Queiroz, A. (2016). Participación social en la gestión ambiental: estudio de caso en una unidad de conservación urbana en el municipio de São Pablo, Brasil. Estudios Demográficos y Urbanos, 31(1), 87-113. https://bit.ly/2SsCkct

Clayton, S. (2019). Psicología y cambio climático. Papeles del Psicólogo, 40(3), 167-173. https://doi.org/10.23923/pap.psicol2019.2902

Corona-Jiménez, M. A. (2018). El conocimiento, la percepción y disponibilidad para afrontar el Cambio Climático en una población emergente, los migrantes de retorno. Estudios Sociales, 28(52), 1-28. https://doi.org/10.24836/es.v28i52.578

Cruz, N. y Páramo, P. (2020). Educación para la mitigación y adaptación al cambio climático en América Latina. Educación y Educadores, 23(3), 469-489. https://doi.org/10.5294/ edu.2020.23.3.6

Espejel, A. y Flores, A. (2015). Conocimiento y percepción del calentamiento global en jóvenes de bachillerato, Tlaxcala. Revista Mexicana de Ciencias Agrícolas, 6(6), 1277-1290. https://bit.ly/3heN5cC

Fernández, I. (1982). NTP 15: Construcción de una escala de actitudes tipo Likert. Instituto Nacional de Seguridad e Higiene en el Trabajo https://bit.ly/3epFy97

Gädicke, J., Ibarra, P. y Osses, S. (2017). Evaluación de las percepciones medioambientales en estudiantes de enseñanza media de la ciudad de Temuco, Región de La Araucanía. Estudios Pedagógicos, 43(1), 107-121. https://www.redalyc.org/pdf/1735/173553246007.pdf

García, C. (2011). El cambio climático: los aspectos científicos y económicos más relevantes. Nómadas. Revista Crítica de Ciencias Sociales y Jurídicas, 32(4), 5-32. http://dx.doi. org/10.5209/rev NOMA.2011.v32.n4.38052

Gil-Guirado, S. (2017). Deconstruyendo riesgos. Cuatro siglos de cambios en la vulnerabilidad y adaptación a las inundaciones y sequías en Murcia. Revista de Historia Moderna, (35), 308-344. https://doi.org/10.14198/RHM2017.35.09 
González, A. (2016). Programa de educación ambiental sobre cambio climático en la educación formal y no formal. Universidad y Sociedad, 8(3), 99-107. https://rus.ucf.edu.cu/index. $\mathrm{php/rus/article/view/418/pdf}$

Hernández, H. A. y Pascual, A. E. (2018). Validación de un instrumento de investigación para el diseño de una metodología de autoevaluación del sistema de gestión ambiental. Revista de Investigación Agraria y Ambiental, 9(1), 157-164. https://doi. org/10.22490/21456453.2186

Masson-Delmotte, V., Zhai, P., Pörtner, H.-O., Roberts, D. Skea, J., Shukla, P. R., Pirani, A., MoufoumaOkia, W., Péan, C., Pidcock, R., Connors, S., Matthews, J. B. R., Chen, Y., Zhou, X., Gomis, M. I., Lonnoy, E., Maycock, T., Tignor, M. y Waterfield, T. (Eds.). (2018). Calentamiento global de $1,5^{\circ} \mathrm{C}$. Grupo Intergubernamental de Expertos sobre el Cambio Climático. https://bit. $\underline{\text { Iy/3eYOiDZ }}$

Martínez-Soto, J. (2004). Comportamiento proambiental. Una aproximación al estudio del desarrollo sustentable con énfasis en el comportamiento persona-ambiente. Theomai, (99). https://bit.ly/3nWiJgl

Matas, A. (2018). Diseño del formato de escalas tipo Likert: un estado de la cuestión. Revista Electrónica de Investigación Educativa, 20(1), 38-47. https://doi.org/10.24320/ redie.2018.20.1.1347

Miranda, R.G. (2018). Desarrollo y cambio climático. Una mirada desde América Latina. Revista del CESLA, (21), 193-212. https://www.redalyc.org/articulo.oa?id=243358276015

Morillo, D., Abad, F. J., Kreitchmann, R. S., Leenen, I., Hontangas, P. y Ponsoda, V. (2019). The Journey from Likert to Forced-Choice Questionnaires: Evidence of the Invariance of Item Parameters. Journal of Work and Organizational Psychology, 35(2), 75-83. https://doi. org/10.5093/jwop2019a11

Ojeda, L. (2018). Imágenes y datos: así nos afecta el cambio climático. Cumbre climática en Polonia, una oportunidad que no podemos perder. Greenpeace. https://bit.ly/3nQZYes

Olmos, E., González, M. E. y Contreras, M. R. (2013). Percepción de la población frente al cambio climático en áreas naturales protegidas de Baja California Sur, México. Polis, (35). https:// journals.openedition.org/polis/pdf/9158

Olmos-Martínez, E., Arizpe, O., Contreras-Loera, M. R. y Gómez-Cabrera, I. D. (2016). El cambio climático: una perspectiva de género. Opción, 32(13), 1136-1157. https://bit.ly/3eqsBMp

Orozco, M. E., Álvarez, G. y Reyes, M. A. (2020). Aptitud social de la percepción ambiental en el Parque Metropolitano Bicentenario, ciudad de Toluca, México. Revista Urbanismo, (42), 151-175. https://doi.org/10.5354/0717-5051.2020.56964 
Paula-Acosta, C. A., Pérez-López, J. y Sierra-Socorro, J. J. (2019). La educación ambiental con enfoque integrador. Una experiencia en la formación inicial de profesores de matemáticas y física. Revista Electrónica Educare, 23(1), 1-22. http://dx.doi.org/10.15359/ree.23-1.10

Páramo, P. (2017). Reglas proambientales: una alternativa para disminuir la brecha entre el decir-hacer en la educación ambiental. Suma Psicológica, 24(1), 42-58. https://doi. org/10.1016/i.sumpsi.2016.11.001

Quintana, F. (2016). Dinámicas, escalas y dimensiones del cambio climático. Tla-Melaua, 10(41), 180-200. https://bit.ly/33ttCwJ

Reyna-Fabian, M., Espinoza, A., Seingier, G., Ortiz-Lozano, L. y Espejel, I. (2018). De la evaluación ecológica a la socio-ecológica: la vulnerabilidad de los arrecifes de coral ante los factores de estrés asociados al cambio climático. Sociedad y Ambiente, 6(17), 59-92. https://bit. ly/33mgQ3f

Restrepo-Betancur, L. F., Peña-Serna, C. y Martínez-González, M. F. (2019). Cambio climático en la ciudad de Medellín-Colombia, en un periodo de cincuenta años (1960-2010). Revista DYNA, 86(209), 312-318. https://doi.org/10.15446/dyna.v86n209.69531

Rodríguez-Rodríguez, J. y Renguant-Álvarez, M. (2020). Calcular la fiabilidad de un cuestionario o escala mediante el SPSS: el coeficiente de alfa de Cronbach. REIRE Revista d'Innovació $i$ Recerca en Educació, 13(2), 1-13. https://doi.org/10.1344/reire2020.13.230048

Ruiz, C. (2013). Instrumentos y técnicas de investigación educativa (3. ${ }^{\text {a ed. }) . ~ D A N A G A ~ T r a i n i n g ~ a n d ~}$ Consulting.

Simms, L. J., Zelazny, K., Williams, T. F. y Bernstein, L. (2019). Does the number of response options matter? Psychometric perspectives using personality questionnaire data. Psychological Assessment, 31(4), 557-566. https://psycnet.apa.org/doi/10.1037/pas0000648

Soares, D., García, A. y Manzano, L. R. (2018). Cambio climático. Percepciones sobre manifestaciones, causas e impactos en el Distrito de Temporal Tecnificado MargaritasComitán, Chiapas. Ciencia ergo-sum, 25(1), e1. https://doi.org/10.30878/ces.v25n1a1

Vargas, L. M. (1994). Sobre el concepto de percepción. Alteridades, 4(8), 47-53. https://bit. $\underline{\text { Iy/3VLIUJn }}$

Vázquez-Guzmán, M. A. (2018). Diseño, validación y aplicación de un instrumento de evaluación de las competencias profesionales para realizar las funciones esenciales de la salud pública. Revista de la Fundación Educación Médica, 21(5), 247-254. https://bit.ly/3hf2ARQ

\section{AGRADECIMIENTOS}

Se agradece la participación de Diana Hernández Hernández y Akemi Aguirre Muñoz, estudiantes del sexto semestre de la carrera de Técnico en Programación, del Centro de Bachillerato Tecnológico, Industrial y de Servicios 166, quienes apoyaron en la aplicación de la encuesta. 